\title{
An Adaptive e-Health Solution System for Disaster Management
}

\author{
N. J. Kulkarni' ${ }^{1}$, Dr. J. W. Bakal ${ }^{2}$ \\ ${ }^{1}$ Research Scholar, Department of Computer Engineering, TSEC, Bandra, Mumbai, India \\ ${ }^{2}$ Research Guide, S.S. Johndhale COE, Dombivali, Thane, Dombivli (East), Thane- 421204, India
}

\begin{abstract}
E-Health monitoring systems provide very good means for progressive monitoring and referential analysis based on historic data measurement \& as well as general reference data of the individuals \& patients, stored in the system. With the advent of Internet of Things (IOT) [1] and increased research and advances in sensor networks for intrusive and nonintrusive health monitoring systems, this will result in an explosion of information across the verticals of e-health to be processed and acted-upon and as IOT matures and with advent of smart environments (smart CARs, smart Homes, smart Hospitals, smart Cities, etc.) this is only going to increase resulting into humongous data being made available to process and analyze - imagine billions of people connected to the network constantly being monitored, their health inputs received \& processed by the systems serviced by few hundred thousands of doctors and every critical alert monitored, tracked \& advised upon. The world average of doctor to patient ratio being 1.6 per 1000 people [3] (varies per country but this is the average calculated for across the globe), this would mean a lot of information \& patients for doctors to act-on simultaneously during a major catastrophic event or disaster impacting multiple individuals, ultimately resulting in a very promising \& a convenient solution having too many practical limitations - either increased over-head due to continuous information would result in medical community becoming less "sensitive" to patient's alerts over-the-time or patients becoming over-caring \& overly-worried or patients losing faith in the e-Health solution \& monitoring system where the technology is seen more of a burden and a cost over-head. In near future, the sensor network are going to play major role in monitoring of the individuals - this will be influenced by rising health costs and healthcare spending which may result into multiple health insurance companies into exploring options like involving smart sensors (intrusive and nonintrusive) to monitor individual's health, habits and habitat. In this paper we attempt to explore the possible architecture that can be considered for adaptive \& early processing of information during a major catastrophic event such natural calamities \& disasters, terrorist attacks, etc. causing impact to multiple human beings. We should be able to successfully identify impacted individuals, direct the critical and fatally wounded/impacted individuals to relevant centers where they can be immediately treated and make this solution have practical approach, result in reduced over-head of information for medical practitioners and help increase the quality of human life.
\end{abstract}

Keywords: Sensor Networks, Adaptive e- Health Monitoring, Internet of Things, Cloud Computing in e-Health, e-Health Disaster Management

\section{Introduction}

The e-Health [2] field is characterized with type of applications, contexts \& theory in which it is used. In near future, the sensor network are going to play major role in monitoring of the individuals - this will be influenced by rising health costs and healthcare spending which may result into multiple health insurance companies into exploring options like involving smart sensors (intrusive and nonintrusive) to monitor individual's health, habits and habitat from a very early stage which may begin as early as from when a person is born. With this view, we started our research in e-Health field to understand how sensor networks (wireless and/or wired) when applied to patient's collection of vital stats intrusively and / or nonintrusively across the environments that is when patient is stationary (at home or hospital) or mobile (car or public transport or any transport) can influence the readings, their interpretation and the over-all therapeutic use of the data. Our research was to enable systems to have a framework that would provide anonymity, confidentiality and thus the security to be considered first and to build the system around this rather than to integrate security into the system at a later stage however along with this another interesting aspect that surfaced was data analytics and systemic behavior during major critical events where majority of the patients would be in emergency situation. Initial study of how e-Health Solution Systems collate and analyze data revolved around various sensors, the protocols to collect the data \& send processed information to medical practitioners to action. There seems to very little study available to understand in depth how the systems will behave in emergency that is few patients have emergency Vs how system will behave in "emergency" when large catastrophic events occur ( for example Earth Quakes, Storms, Terrorist Attacks, WAR, etc.) Vs how the system will behave when multiple human resources engaged in support of e-Health (medical practitioners, nurses, and ambulances) are already busy handling multiple critical patients. This would mean the entire e-Health solution infrastructure (software \& hardware infrastructure, communication network, medical support fraternity) will be under complete stress and collapse or crash. As per 2009 data collection statistics India has a ratio of 0.65 Doctors / 1000 people, United States 2.42 (as per 2009 data), United Kingdom 2.77 ( as per 2011 data), Germany 3.69 ( as per 2010 data), Australia 3.85 ( as per 2010 data) and this keeps varying while many developing \& African nations across globe dwell in 0.6 to 0.02 even [4] To add into this the world average of doctor's availability is 1.6 per 1000 people [3]. This will also mean that patient will not receive the timely availability of proper medical services and above all the medical fraternity will be completely scattered with priorities, alerts will which will be provided by the system will be over-looked as physical resources are not available and the very the reason the e-health solution was created will fail and we will find ourselves facing the 


\section{International Journal of Science and Research (IJSR) \\ ISSN (Online): 2319-7064}

Index Copernicus Value (2013): 6.14 | Impact Factor (2014): 5.611

same hard and ugly issues, which we had the designed the solution to address for us, in the first place. Delayed emergency responses, failure in co-ordination \& management was characterized in catastrophes like Haiti earthquake resulted in deaths of 160,000 people [7] with estimated number of injured ranging between 200,000 to 300,000 [6], in 2011 the tohoku earthquake resulted in the death of 15,889 people, 6152 injured and 2601 missing [7], in 2004 Indian Ocean tsunami more than 280,000 people died in a multiple countries [5] with $\sim 125,000$ injured and other $\sim 45,000$ missing.

\section{2. e-Health Patient Monitoring System}

Consider an e-Health Solution system that transmits continuous sensory data collected from individuals (monitored) by intrusive and nonintrusive sensory equipment. As shown in Fig. 1 below, the sensory data is transmitted directly to Service Provider's cloud or through local gateway or patient's PDA or smart phone.

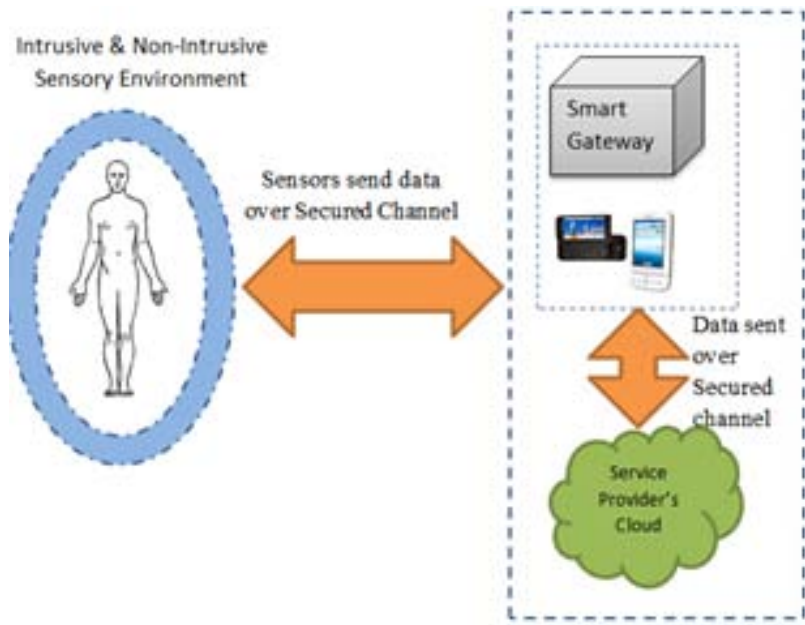

Figure 1: Sensor Data Collection and secured transmission to Service Provider's Cloud

The sensor's data is received by the e-Health Service provider's cloud where it collated \& processed. After analyzing these readings \& understanding any peaks \& offsets that may influence the over-all health of the individual, special algorithms using deterministic \& probability arrive at best case estimation of the symptomatic $\&$ therapeutic nature of the ailment/disease/symptom and accordingly take actions based on priority. The Fig. 2 provides details of how the algorithms once on service provider's cloud act on the data provide related support:

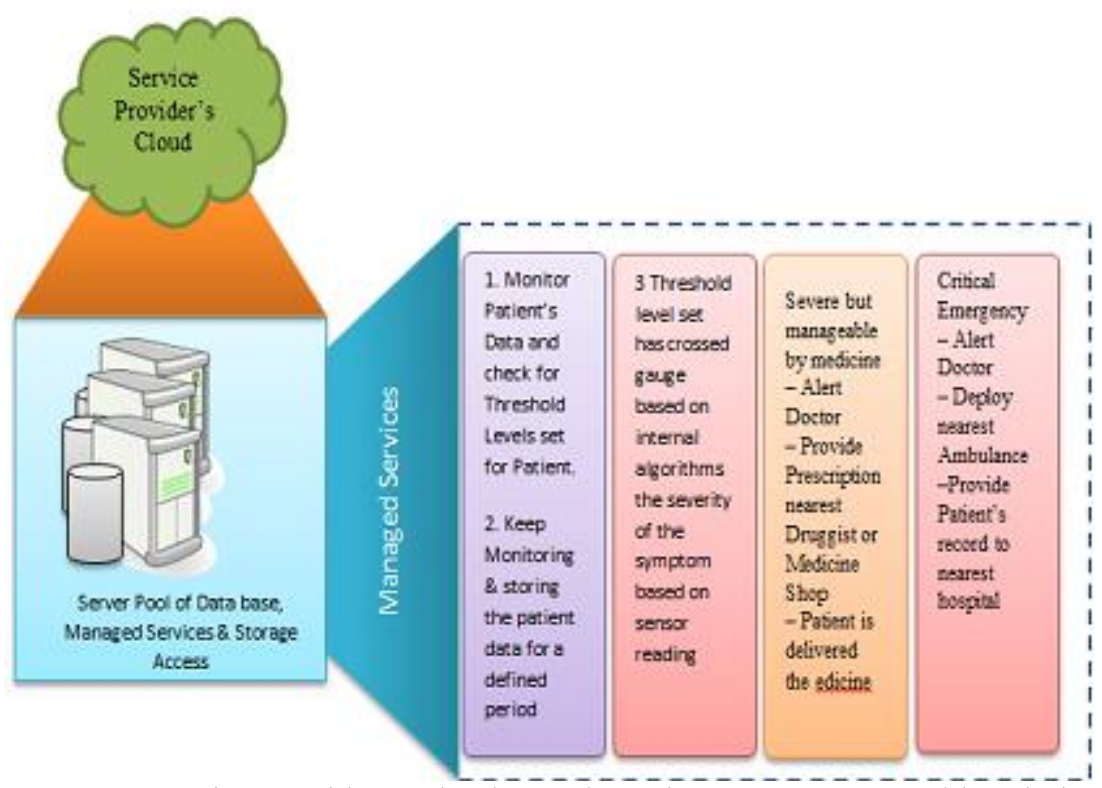

Figure 2: Service Provider's Cloud Based Services manages e-Health Solution

The e-Health System identifies the critical or emergency situation and accordingly initiates an action that includes immediate assistance by medical practitioner or delivery of medicines by nearby pharmacy or medical stores or sending ambulance \& admitting the patient to the correct hospital with all historical in fsformation \& reports provided to hospital for early and proper treatment.

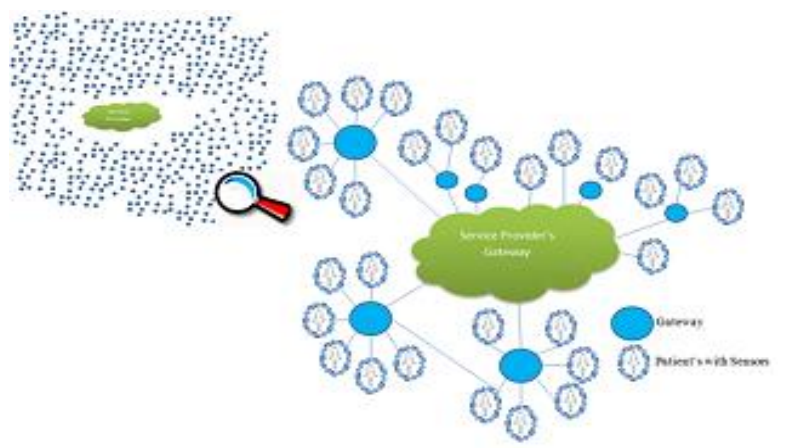

Figure 3: Sensors Network communicating with Service Provider's Gateway

\section{Volume 5 Issue 1, January 2016 www.ijsr.net}




\section{International Journal of Science and Research (IJSR) \\ ISSN (Online): 2319-7064}

Index Copernicus Value (2013): 6.14 | Impact Factor (2014): 5.611

As seen in Fig. 3, the system deals with an extensively large base of individuals being monitored for e-Health program. Now consider a large scale event (natural disaster or some calamity or catastrophe) where a "mass" number of individuals are impacted simultaneously. Thus multiple individuals being monitored in the impacted situation will start reporting alerts emergency \& critical care. In general following observations based on systemic behavior can be made inability to get real-time inputs as sensor network based on type of disaster may get impacted, damaged sensors, ambulances deployed but unable to identify and help individuals with life threating symptoms, doctors shuffling between patients without access to proper data, once network is restored flood of critical alerts provided by the sensors to the system and then to individual hospitals, multiple simultaneous reports accessed \& requested by hospitals \& system, etc. Thus, the result is again a massive chaos \& confusion and even though system was initially designed \& implemented to handle this very situation. To add into this doctors and medical infrastructure will remain under constant pressure to deal with multiple patients. The challenges are multiple and only few are listed above. Thus we understand that an advance analytical engine with ability to communicate only the necessary information [8] with minimal transmissions in a securely manner, ability for a guaranteed communication network infrastructure at multiple levels is required so that we can provide more advised inputs to medical fraternity and adapt to compute, network \& systemic requirements based on real-time inputs and proactively provide "next steps" or intuitive information rather than provides "inputs" based on data requested for access.

We can thus chalk out following characteristic necessary for the architecture of adaptive e-Health Solution:

- Scalability: Integration issues of different sensors \& heterogeneous networks due to proprietary development.

- Security, Anonymity \& Confidentiality: The system should be accessible by concerned \& authorized personnel only. Confidentiality \& anonymity wherever applicable is the key to avoid misuse and gain end-user faith in the system. Current model of rely on existing security infrastructure may not be effective \& successful in maintaining secured access \& storage.

- Disaster \& Catastrophe: Information over-head, multiple priorities, successful identification of individuals, hospital management, and patient data management should be effectively managed based on real-time inputs.

- Communication Network: Alternate e-Health Emergency \& Remote Availability Network, Emergency Network Modules, Alternate E-Health communication channel for emergency \& remote places, network pods and emergency relay drones need to be available to relay patient \& patient's information in real-time.

- Medico-Legal: Ability to successfully quantify \& qualify medical practitioner's decision based on actual inputs. Hence secured access, data retention, access privileges, etc would be mandatory.

\section{Proposed System}

The proposed system has multiple levels and design aspects to be considered right from security aspect to way the sensors and sensor network will integrate with the system providing security \& ensuring confidentiality. However, in this paper we are emphasizing on how the proposed system will effectively handle the scenario described above. At the time of disaster or any crisis that impacts or influences multiple individuals a pattern would be observed. As shown in Fig. 4 - A, a section of monitored individuals from a geography or a location will stop reporting all data as communication network in that area has been impacted or as shown in Fig. 4 - B, once network is made available in that impacted geography by use of temporary public e-Health Hotspot or other means all the individual's sensors would start transmitting massive amounts of critical alerts based on individual's health conditions.

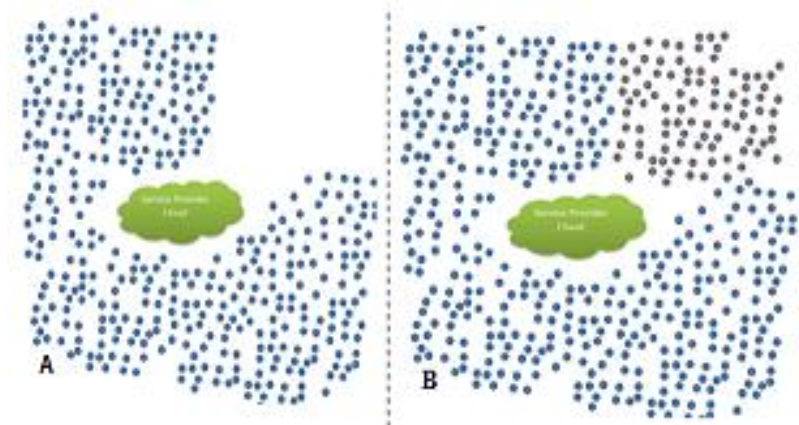

Figure 4: B displays impacted location with no-alerts or communication from that location \& Figure 4-B shows communication alerts being sent by impacted individuals.

The system (on service provider's cloud) monitors the weather and any signs for disasters or events in areas of interest over the internet as well as keeps monitoring for patterns (viz. sudden burst of alerts from a locality or plurality of areas or missing alerts or no data about individuals being monitored from a locality or plurality of areas) as reported above. The architecture thus in a disaster scenario relies on internal algorithms to proactively collate data from across multiple individuals, hospitals, medical practitioners, disaster information and patterns, restored communication network based on real-time inputs. In event of an emergency:

\section{A. Sensor Network to Cloud}

It is possible that during disaster, the communication network may get hampered and require alternate communication network to be established to send sensor's data to e-Health's cloud. Thus the sensor network should have the ability to connect with Emergency Communication Network or temporary Public Medical Hotspots \& relay "health" specific information only in a secured \& confidential manner. Ideally sensors communicate with gateway, smart phones or cloud in a secured unicast manner over-a-secured channel. However the same model cannot be applicable during "individual's" emergency or when local communication gateway is not available. Thus the sensor network transitions transmission of data to broadcast domain on a well-known set of ports. The sensors networks thus work in dual modes private \& known secured gateway, public medical hotspot device-agents. 


\section{International Journal of Science and Research (IJSR) \\ ISSN (Online): 2319-7064}

Index Copernicus Value (2013): 6.14 | Impact Factor (2014): 5.611

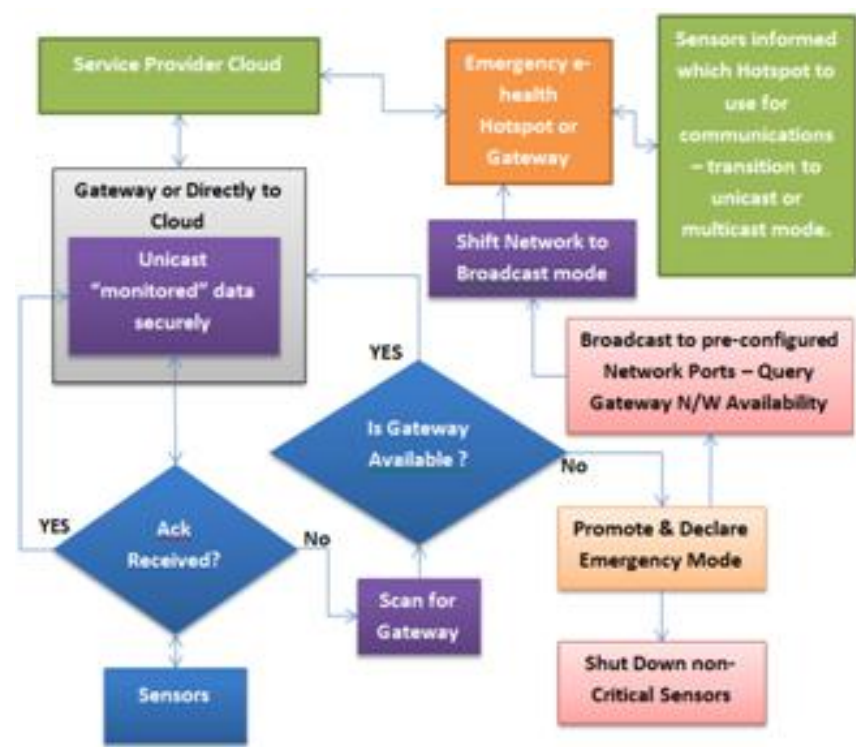

Figure 5: Sensor Network in absence of gateway will search for e-Health Hotspots by transitioning to Broadcast domain.

Once network is established and /or available for sensor network to communicate with e-Health Cloud it then works on the continuously available data \& Alerts and processes them. The proposed architecture proposes definition \& availability of additional communication channel for health network on broadcast domain.

\section{B. Cloud Engine to Systematically Handle Crisis:}

The cloud engine has special algorithms that actively scans $\&$ updates the database based on real-time inputs about location, locality and availability of multiple data sets. It maintains a very categorical database about the availability of number of doctors across the hospitals and their specialty (so patient with internal bleeding having diabetes is treated by doctor who has specialty with dealing such patients), different hospitals in nearby vicinity of affected area, ambulances and emergency services, hospitals, blood banks, list of active donors of blood (with rare \& regular), Hospitals with Specialties, information of ICU \& bed availability, infrastructure availability, support staff \& doctor's availability in real-time for which it keeps tab on hospital inputs. It also maintains a detailed list of emergency workers trained in First Aid, Nurses, Physicians, etc.

The algorithms check the critical alert messages received for the patients. Every patient at the time of registration with the e-Health Solution will have his biometrics registered with the system. Alerts are categorized as life threating and nonlife threating and accordingly information about each patient is collated \& categorized. This information is then relayed to every emergency personnel's hand held device. The information is about biometrics to identify patients, their severity and directions about whether the patient is critical as per sensor data or not and to which hospital the patient needs to be taken for treatment. Since the engine understands certain sensors on individuals may have malfunctioned, every individual's data along with best case determination as to which hospital the person can get best treatment is gauged \& readily provided on the hand held device. On field the patient identification takes place each individual's based on biometrics (finger (hand/legs/retina) or id on individual the patient is identified. Once the patient is identified (should not take more than 1 to 30 seconds) the Emergency person's hand held device reflects data that helps him provide any immediate precautionary measures to stabilize the patient, details of address of the Hospital where patient needs to be taken.

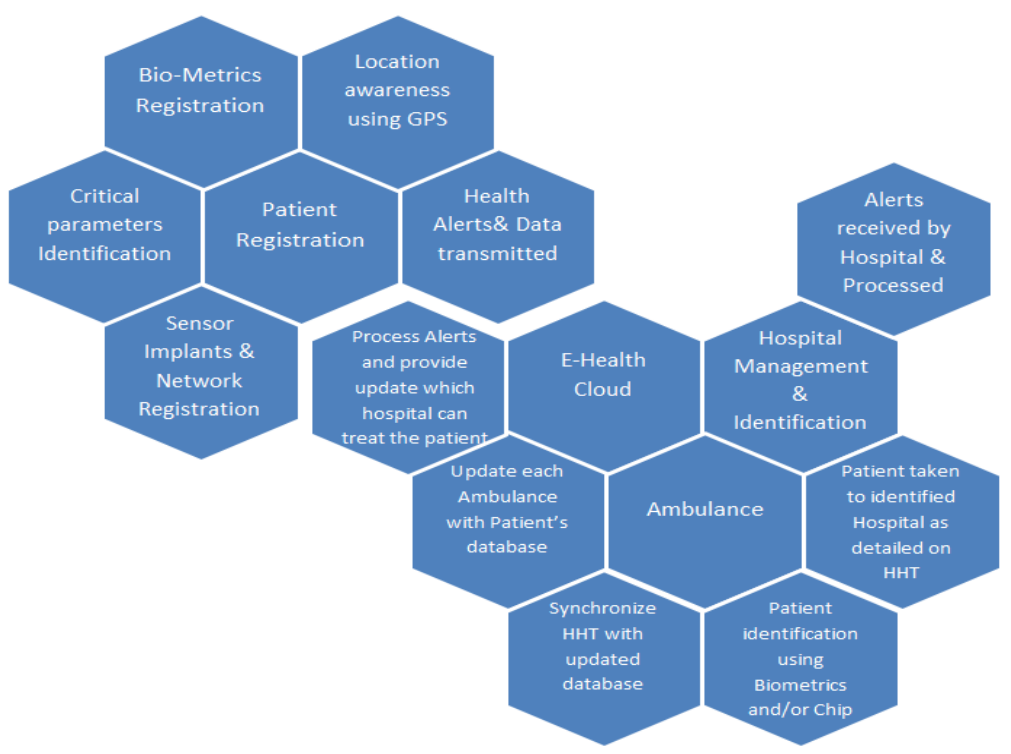

Figure: A Module based view of the solution that will be extremely active during Disaster Scenario

E-Health's cloud engine would receive critical alerts for immediate medical attention from multiple patients. Sensors trigger appropriate alerts; some sensors may have malfunctioned or be sending corrupt data. All those alerts are collated by the system \& processed (those identified invalid or noncritical are discarded). Once the emergency person identifies \& tags the individual as a patient and needs immediate medical assistance a flag is raised. The sensors continuously relay alerts to Cloud Engine which are further relayed to correct hospitals based on selection criteria. The

\section{Volume 5 Issue 1, January 2016}




\section{International Journal of Science and Research (IJSR) \\ ISSN (Online): 2319-7064 \\ Index Copernicus Value (2013): 6.14 | Impact Factor (2014): 5.611}

hospitals do have the ability to reduce the frequency of alerts received by them based on the availability, which they keep on updating in the system. The algorithms within the cloud engine are capable enough to scale and cope up with any emergency as well as adapt and take decisions based on realtime inputs. Since the system knows where any patient or individual is being treated or getting medical assistance, the relatives or emergency contacts of those individuals can be provided accurate information about the patient and their location. Also the ability of the system to provide specific inputs to doctors along with historic data help the patient to get correct timely treatment. The cloud engine also ensures that the information received and requested by doctors/physicians while treating the patients is very specific. A doctor who may be seeing the individual for the first time, has no medical history of the patient and has to operate on the patient and he/she then needs to hop to next patient should be able to make advised decisions very quickly. The initial reports generated contain very specific information related allergies \& medical conditions. The historic data is only provided when requested by the Doctor. The information is sufficient so that the doctor can immediately start patient's treatment by going through it within a minute or two.

\section{Conclusion}

The adoption of e-Health System to monitor individual's health will soon become a necessity and in near future rising costs of healthcare may necessitate remote monitoring of individual health as mandatory requirement by interested parties. In such a situation where multiple individuals are being monitored, should there be any disaster or catastrophe resulting in a mass damage to human lives \& infrastructure there will be extreme stress on all the resources be it the compute, communication networks, hospitals and medical community. The proposed e-Health System is designed with ability to handle disaster scenarios effectively, thus is successful even in the most chaotic situations with successfully identifying the identity of critically injured or wounded or unconscious persons using biometrics as well as smart chips and alerts the emergency personnel attending the patient on disaster site which nearest hospital will be able to provide the correct treatment to the patient which includes synchronizing movement of critical assets like ICU bed availability if required, checking \& ensuring availability of blood (rare groups includes) to be available at the hospital, along with vital information like blood group, allergens details updated to concerned hospital(s). The system on realtime basis accepts inputs from various hospitals on beds available, doctors available and also is capable of mobilizing resources for emergency medicines to be available to the various hospitals \& institutions based on real-time-inputs of requirements. During any major disaster or catastrophe it is highly likely that the communication infrastructure may have been impacted. The architecture takes into consideration this possibility and entire sensor network is managed in priority \& check availability model. The security of sensor data is maintained as the temporary EHealth Hotspots (made available by communication drones, ballons or mobile network VANs) listen for broadcast and then subsequent multicasts on specified ports and do not attempt to analyze any data, instead they transmit the data to the E-health's cloud engine where the sensors data is processed \& only the required \& necessary actions are relayed to the ambulance's computer which are further synchronized on emergency personnel hand held terminals which aid with identifying the patients \& necessary procedures to immediately tackle any emergency and details of nearest hospital that can treat the patient. In this manner we are also able to establish an immediate network which ignores cellular or data traffic and concentrates on e-Health Sensor traffic, is able to identify the individuals during emergency, provide inputs where patients get immediate treatments the relatives, near or dear-ones are also immediately updated on where the individual is on real-time (last known location) which hospital and what treatment is being given. This will also help families to find the near \& dear ones almost immediately without running from pillar to-post to understand where individual they are searching for is located. Also during emergency, doctors are pulled in on treating multiple patients, as the patient's history (blood group, medical history including allergy, etc.) is immediately available to doctors they can immediately start treatment to patients and can treat multiple patients. The system also makes available the entire medical history to concerned registered medical practitioner or hospital authorities only if the person is admitted to that hospital, there-by maintaining certain amount of confidentiality that personal sensitive information is not available to everyone. The algorithm also works in tandem to provide information that will aid the medico-legal decisions in a manner that supports the data to consider such decisions using probability \& deterministic models rather than simply provide inputs \& results that can have multiple interpretations.

\section{References}

[1] Xu Li, Rongxing Lu, Xiaohui Liang, and Xuemin (Sherman) Shen, University of Waterloo,Jiming Chen Zhejiang University, Xiaodong Lin, University of Ontario Institute of Technology "Smart Community: An Internet of Things Applications", pp. 68-75, IEEE Communications Magazine, November 2011.

[2] Monika Grajzer, Piotr Szczechowiak, " eHealth Traffic Detection and Classification Using Machine Learning Techniques", pp.151-154, The Fourth International Conference on eHealth, Telemedicine, and Social Medicine, ISBN: 978-1-61208-179-3, 2012.

[3] Calculated average ratio based on the summation of all ratios across globe provide by https://www.cia.gov/library/publications/the-worldfactbook/fields/2226.html

[4] http://data.worldbank.org/indicator/SH.MED.PHYS.ZS

[5] http://www.who.int/hac/crises/international/asia_tsunam i/3months/report/en/

[6]

http://en.wikipedia.org/wiki/Casualties_of_the_2010_H aiti_earthquake

[7] https://www.pinterest.com/w15692000/tohoku-afterthe-311-earthquake-and-tsunami/

[8] A Prehospital Database System For Emergency Medical Services, Chapter XIV, Unwired Business: Cases in Mobile Business edited by Stuart Barnes and Eusebio Scornavacca (C) 2006, Idea Group Inc.

\section{Volume 5 Issue 1, January 2016

